\title{
A MODEL FOR A DYNAMIC PREVENTIVE MAINTENANCE POLICY
}

\author{
CHRISTIANE COCOZZA-THIVENT \\ Université de Marne-la-Vallée \\ Equipe d'Analyse et de Mathématiques Appliquées \\ Cité Descartes, 5 Boulevard Descartes, Champs-sur-Marne \\ 77454 Marne-la-Vallée Cedex 2, France
}

(Received August, 1999; Revised June, 2000)

This paper exhibits a stochastic model which describes the evolution of a material submitted to inspections. When an inspection takes place, a decision depending on the observed state of the material is taken. If the material is in "not too bad" state, no service is rendered, only the date of the next inspection is chosen. If the material is in a "bad" working state, a service takes place. Roughly speaking, the failure rates of the material are constant, the inspection and repair rates are general. We define the average cost function corresponding to the utilization of this material and we show how it can be computed. Then we determine the inspection rates which give the optimal maintenance policy using a simulated annealing algorithm. We observe experimentally that the best durations between inspections are deterministic ones.

Key words: Preventative Maintenance, Conditional Maintenance, Cost Function, General Repair Rate, Stationary Distribution, Optimization, Optimal Maintenance Policy, Simulated Annealing Algorithm, Supplementary Variables.

AMS subject classifications: 60J05, 60J25, 60J75, 60K10, 60K15, $60 \mathrm{~K} 20$.

\section{Model Introduction}

In [4], P.A. Scarf discusses the opportunity of developing areas of maintenance modeling. Among special growth areas, he mentions the dynamic maintenance policies for complex systems and remarks that, developing dynamic policies is significantly more difficult than developing static policies.

Here we present such a model for systems that can be described by a finite number of states. In [3], the authors are interested in the same kind of problem, but the evolution of the systems they consider can be represented by the evolution of a real parameter. 
Our model can be roughly described as follows. Let us first suppose that no inspection is planned. The material we are studying degrades progressively until it reaches a failure state, the transitions from working states to other working states or to failure states being constant. Let us now add inspections. When an inspection takes place, if the material is observed in a state which is not too damaged, no service is rendered and the date of the next inspection is chosen according to a distribution which depends on the observed state. If the material is observed in a too damaged state, a service is rendered. The repair, service and inspection rates are general (they are not supposed to be constant).

Our aim is to compute an average cost corresponding to the utilization of the material over an infinite period. In order to study the asymptotic system, we introduce supplementary variables for obtaining a Markov process. We show that the average cost can be expressed using the stationary distribution of this Markov process. The computation of this stationary distribution leads to a system of differential equations that are explicitly solved. The initial conditions of this system are solutions to a linear system of equations.

Formulas show that usually cost depends on the average of repair and service durations, but does not depend on the shape of their distribution functions. On the other hand, cost does depend on the shape of the distribution functions of the durations between inspections.

We give numerical examples, then we optimize the maintenance policy using a simulated annealing algorithm. We observe experimentally that the best durations between inspections are deterministic ones.

Let us now describe our model more explicitly.

\subsection{The Initial Model}

Let us consider a material (called "system" for the time being) for which the evolution of time is described by a stochastic process with values in a finite space $E$. The elements of $E$ are denoted by Greek letters: $\eta, \xi, \zeta, \ldots$. The subsets of working and failure states are denoted by $\mathcal{H}$ and $\mathscr{P}$, respectively.

As time goes on, the material degrades progressively until it reaches a failure state (this idea of degradation is not essential for what follows, but it allows one to easily represent the evolution).

While the material is working, its evolution is Markovian. When the material breaks down, it is repaired. The rate of repair depends on the time, on the failure state $\eta \in \mathscr{P}$ of the material, and on the state $\xi \in \mathcal{M}_{\mathrm{g}}$ in which the material will be at the end of the repair. It is denoted by $\mu(\eta, \xi, x)$.

Let $A_{1}$ be the matrix for which the nondiagonal elements are the transition rates between the working states. Let $A_{12}$ be the matrix of the transition rates between the working states and failure states and $A$ be the matrix for which the nondiagonal elements are the transition rates from the working states: $A=\left(A_{1} A_{12}\right)$. The diagonal entries of $A$ and $A_{1}$ are given by

$$
\forall \eta \in \mathcal{H}, \quad A_{1}(\eta, \eta)=A(\eta, \eta)=-\sum_{\substack{\xi \in E \\ \xi \neq \eta}} A(\eta, \xi) .
$$


The sum of terms of each line of $A$ is therefore zero.

Remark 1: Let us denote by $A^{\prime}$ the matrix $A$ to which we add a number card ( $\mathscr{P}$ ) lines full of zeros in order to have a square matrix. The matrix $A^{\prime}$ is the generator matrix of the Markov jump process described above for which, moreover, the failure states are absorbent.

\subsection{The Preventive Maintenance Model}

The system is also submitted to inspections to carry out servicing called preventive maintenance operations for the time being. Let $\left(\mathcal{M}_{g}, \mathcal{M}_{\mathcal{B}}\right)$ be a partition of the set of working states in two finite subsets. When an inspection takes place, if the material is in the state $\eta$ belonging to $\mathcal{M}_{\mathrm{g}}$, no service is rendered and the length of time until the next inspection is chosen according to a distribution dependent on $\eta$. If during an inspection, the material is in a state belonging to $\mathcal{M}_{\mathcal{E}}$, a service takes place and at the end of this service, the material is in a state belonging to $\mathrm{Mog}$.

A label $i=\ell(\eta)$ is associated with each $\eta \in \mathcal{M}_{\mathrm{g}}$, and a rate $\Lambda_{i}$ is associated with each label $i$. This rate $\Lambda_{i}$ is the hazard rate corresponding to the duration of the period of time which elapses before a next inspection will take place. The p.d.f. of the duration of this period is therefore:

$$
\Lambda_{i}(x) e^{-\int_{0}^{x} \Lambda_{i}(u) d u}
$$

The introduction of the function $\ell$ is logically unnecessary, especially if we assume it to be bijective (whereas no hypothesis is made on the function $i \rightarrow \Lambda_{i}$ ), but it allows a greater ease in reading the results. It allows to discriminate between what corresponds to a current state of the system and what corresponds to an inspection label.

If at the initial time, the material is in state $\eta \in \mathcal{M}_{\mathrm{g}}$, the next service will take place after a length of time whose rate is $\Lambda_{\ell(\eta)}$. Similarly, when the material comes back to the state $\xi$ following a service or repair, the next service will take place after a length of time whose rate is $\Lambda_{\ell(\xi)}$.

A service, which takes place when the material is in a state $\eta \in \mathcal{M}_{g}$, puts the material in the state $\varphi(\eta)$. The transition rate from state $\varphi(\eta)$ to state $\xi \in \mathcal{N}_{\mathrm{g}}$ is $\mu(\varphi(\eta), \xi, x)$.

We denote by $\&=\left\{\varphi(\eta): \eta \in \mathcal{M}_{g}\right\}$ the set of states corresponding to service and $E_{1}=E \cup 8$.

The objective of this article is to determine the average cost over an infinite period given:

- the cost of an inspection (cost of displacement),

- the cost of parts,

- the hourly cost of labor,

- the cost of the immobilization of material, distinguishing between the predicted immobilization (due to service) and unpredicted immobilization due to failure.

\section{Stationary Distribution}

Let us denote $\Phi_{t}$ the state of the system at time $t\left(\Phi_{t} \in E_{1}\right), I_{t}$ the value of the label at time $t$. The label $I_{t}$ is constant between two inspections. When an inspection 
does not lead to service, it takes the value $i=\ell(\eta)$ of the label associated with the state $\eta$ in which the material finds itself when inspected. Lastly, it takes the value 0 during a service or repair. We assume that $0 \notin\left\{i=\ell(\eta): \eta \in \mathcal{M}_{\mathrm{g}}\right\}$. The set of values taken by the labels is annoted $\mathcal{L}(\mathcal{L}=\{i=\ell(\eta): \eta \in \mathcal{N}\} \cup\{0\})$.

The process $\left(\Phi_{t}, I_{t}\right)$ is not a Markov process. In order that it becomes a Markov process, we add a supplementary variable $X_{t} \in \mathbb{R}_{+}$. If $\Phi_{t} \in \mathcal{M}, X_{t}$ is the time passed since the last inspection. If $\Phi_{t} \in \mathscr{P}$ (respectively, $\Phi_{t} \in \mathbb{8}$ ), $X_{t}$ is the time passed since the start of repair (respectively, service) in progress.

We assume that the functions $\Lambda_{i}(i \in \mathcal{L})$ and $\mu$ are continuous and bounded.

The process $\left(\Phi_{t}, I_{t}, X_{t}\right)$ is a Markov process. By taking the same type of proof as in [2], we can show that its infinitesimal generator $L$ is given, for any function $f$ defined on $E_{1} \times \mathcal{L} \times \mathbb{R}+$, taking values in $\mathbb{R}$, and having a continuous derivative with respect to the third variable, by:

$$
\begin{aligned}
& (L f)(\zeta, j, x)=1_{\{\zeta \in \mathcal{M}\}} \sum_{\substack{\xi \in \mathcal{N} \\
\xi \neq \zeta}} A(\zeta, \xi)[f(\xi, j, x)-f(\zeta, j, x)] \\
& +1_{\{\zeta \in \mathcal{M}\}} \sum_{\xi \in \mathscr{\rho}} A(\zeta, \xi)[f(\xi, 0,0)-f(\zeta, j, x)] \\
& +1_{\{\zeta \in \mathscr{P} \cup \xi\}} \sum_{\xi \in \mathcal{M}_{g}} \mu(\zeta, \xi, x)[f(\xi, \ell(\xi), 0)-f(\zeta, j, x)] \\
& +1_{\left\{\zeta \in \mathcal{M}_{g}\right\}} \Lambda_{j}(x)[f(\zeta, \ell(\zeta), 0)-f(\zeta, j, x)] \\
& +1_{\left\{\zeta \in \mathcal{M}_{\mathrm{g}}\right\}} \Lambda_{j}(x)[f(\varphi(\zeta), 0,0)-f(\zeta, j, x)]+\frac{\partial f}{\partial x}(\zeta, j, x) .
\end{aligned}
$$

We will show in the appendix that, under suitable conditions of irreducibility, there exists a regeneration state $\eta_{r} \in \mathscr{S} \cup \&$ such that for any $(\eta, i, x) \in E_{1} \times \mathcal{L} \times \mathbb{R}_{+}$, $\mathbb{P}_{(\eta, i, x)}\left(\exists t: \Phi_{t}=\eta_{r}\right)=1$. Consequently, for any non-negative bounded measurable function $f$ defined on $E_{1} \times \mathcal{L} \times \mathbb{R}_{+}$, the function $t \rightarrow \mathbb{E}\left(f\left(\Phi_{t}, X_{t}, I_{t}\right)\right)$ verifies a renewal equation. Theorem 2 of [5] therefore allows us to show that $\mathbb{E}\left(f\left(\Phi_{t}, X_{t}, I_{t}\right)\right)$ converges when $t$ tends to infinity to $\int f d \Pi$, where $\Pi$ is the stationary distribution of the process $\left(\Phi_{t}, I_{t}, X_{t}\right)$. We will say that the process is ergodic.

We suppose hereafter that the process is ergodic.

We are going to look for a stationary measure which admits a density having a continuous derivative. It means that we are looking for a measure $\Pi$ of the form

$$
\Pi f=\sum_{\eta \in E_{1}} \sum_{i \in \mathcal{L}} \int_{0}^{+\infty} f(\eta, i, x) \pi(\eta, i, x) d x,
$$

where functions $x \rightarrow \pi(\eta, i, x)$ have continuous derivatives. We will, by writing the stationarity condition $\Pi L=0$, deduce the expression for $\pi(\eta, i, x)$.

For $\eta \in \mathcal{M}$, let us define

$$
q(\eta)=|A(\eta, \eta)|=\sum_{\substack{\xi \in E \\ \xi \neq \eta}} A(\eta, \xi)
$$


and for $\eta \in E_{1}, x \in \mathbb{R}_{+}$,

$$
\bar{\pi}(\eta, x)=\sum_{i \in \mathcal{L}} \pi(\eta, i, x), \pi(\eta)=\int_{0}^{+\infty} \sum_{i \in \mathcal{L}} \pi(\eta, i, x) d x
$$

and for $\eta \in \mathscr{P} \cup \mathcal{B}$

$$
\bar{\mu}(\eta, x)=\sum_{\xi \in \mathcal{M}_{g}} \mu(\eta, \xi, x) .
$$

We will say that the function $f$ defined on $E_{1} \times \mathcal{L} \times \mathbb{R}_{+}$belongs to the class $T(\eta, i)$ if it can be written:

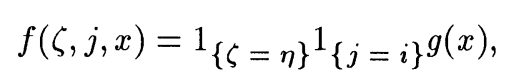

where $g$ is a function defined on $\mathbb{R}_{+}$, with compact support and having a continuous derivative.

Lemma 2: Let $\eta \in \mathcal{H}, i \in \mathcal{L}$ and let us suppose that the function $x \rightarrow \pi(\eta, i, x)$ has a continuous derivative. Equation $\Pi L f=0$ is satisfied for any function $f \in T(\eta, i)$ if and only if:

$$
\begin{gathered}
\frac{d}{d x} \pi(\eta, i, x)=-\left(q(\eta)+\Lambda_{i}(x)\right) \pi(\eta, i, x)+\sum_{\substack{\xi \in \mathcal{M} \\
\xi \neq \eta}} A(\xi, \eta) \pi(\xi, i, x), \\
\pi(\eta, i, 0)= \\
1_{\left\{\eta \in \mathcal{M}_{\mathrm{g}}, \ell(\eta)=i\right\}}\left(\sum_{\zeta \in \mathcal{P}_{\mathcal{O}} \int_{0}} \int_{0}^{+\infty} \mu(\zeta, \eta, x) \bar{\pi}(\zeta, x) d x+\sum_{j \in \mathcal{L}} \int_{0}^{+\infty} \Lambda_{j}(x) \pi(\eta, j, x) d x\right) .
\end{gathered}
$$

Proof: Let $f$ be in $T(\eta, i)$. We have:

$$
\begin{aligned}
& L f(\zeta, j, x)=-q(\eta) 1_{\{\zeta=\eta\}} 1_{\{j=i\}} g(x)+1_{\{\zeta \in \mathcal{M}, \zeta \neq \eta\}} A(\zeta, \eta) 1_{\{j=i\}} g(x) \\
& +1_{\{\zeta \in \mathscr{P} \cup \mathbb{8}\}}{ }^{1_{\left\{\eta \in \mathcal{M}_{g}\right\}}} \mu(\zeta, \eta, x) 1_{\{i=\ell(\eta)\}} g(0)-1_{\{\zeta=\eta\}} 1_{\{j=i\}} \Lambda_{i}(x) g(x) \\
& +1_{\{\zeta=\eta\}} 1_{\left\{\eta \in \mathcal{M}_{g}\right\}} 1_{\{\ell(\eta)=i\}} \Lambda_{j}(x) g(0)+1_{\{\zeta=\eta\}} 1_{\{j=i\}} g^{\prime}(x) .
\end{aligned}
$$

Equation $\Pi L f=0$ is written as:

$$
\begin{gathered}
0=-q(\eta) \int_{0}^{+\infty} \pi(\eta, i, x) g(x) d x+\sum_{\substack{\zeta \in \mathcal{M} \\
\zeta \neq \eta}} A(\zeta, \eta) \int_{0}^{+\infty} \pi(\zeta, i, x) g(x) d x \\
+1_{\left\{\eta \in \mathcal{M}_{g}\right\}} 1_{\{\ell(\eta)=i\}} g(0) \sum_{\zeta \in \Phi \cup 8} \int_{0}^{+\infty} \mu(\zeta, \eta, x) \bar{\pi}(\zeta, x) d x \\
-\int_{0}^{+\infty} \pi(\eta, i, x) \Lambda_{i}(x) g(x) d x
\end{gathered}
$$




$$
+1_{\left\{\eta \in \mathcal{M}_{g}\right\}} 1_{\{\ell(\eta)=i\}} g(0) \sum_{j} \int_{0}^{+\infty} \pi(\eta, j, x) \Lambda_{j}(x) d x+\int_{0}^{+\infty} g^{\prime}(x) \pi(\eta, i, x) d x .
$$

Integrating by parts, we obtain:

$$
\int_{0}^{+\infty} g^{\prime}(x) \pi(\eta, i, x) d x=-g(0) \pi(\eta, i, 0)-\int_{0}^{+\infty} g(x) \frac{d}{d x} \pi(\eta, i, x) d x
$$

We transfer this last version into the former. As the equation obtained is true for any function $g$ with compact support and having a continuous derivative, it remains only to write that the terms which are factors of $g(x)$ (under integral) and those which are factors of $g(0)$ are equal to zero to obtain the claimed result.

By applying the same methodology with the functions $f(\zeta, j, x)=$ $1_{\{\zeta=\eta\}^{1}}{ }_{\{j=i\}} g(x)$, respectively for $\eta \in \mathcal{E}$ and $\eta \in \mathscr{P}$, we obtain the following two lemmas:

Lemma 3: Let $\eta \in \mathcal{E}, i \in \mathcal{L}$ and let us suppose that the function $x \rightarrow \pi(\eta, i, x)$ has a continuous derivative. Equation $\Pi L f=0$ is satisfied for any function $f \in T(\eta, i)$ if and only if

$$
\begin{gathered}
\frac{d}{d x} \pi(\eta, i, x)=-\bar{\mu}(\eta, x) \pi(\eta, i, x) \\
\pi(\eta, i, 0)=1_{\substack{\{i=0\} \\
\zeta \in \mathcal{M}_{\mathbb{E}} \\
\varphi(\zeta)=\eta}} \sum_{j} \int_{0}^{+\infty} \Lambda_{j}(x) \pi(\zeta, j, x) d x .
\end{gathered}
$$

Therefore, in this case, for $i \neq 0, \pi(\eta, i, x)=0$ and

$$
\bar{\pi}(\eta, x)=\pi(\eta, 0, x)=e^{-\int_{0}^{x} \bar{\mu}(\eta, u) d u} \sum_{\substack{\zeta \in \mathcal{M}_{\&} \\ \varphi(\zeta)=\eta}} \sum_{j} \int_{0}^{+\infty} \Lambda_{j}(x) \pi(\zeta, j, x) d x .
$$

Lemma 4: Let $\eta \in \mathscr{P}, i \in \mathcal{L}$ and let us suppose that the function $x \rightarrow \pi(\eta, i, x)$ has a continuous derivative. Equation $\Pi L f=0$ is satisfied for any function $f \in T(\eta, i)$ if and only if:

$$
\begin{gathered}
\frac{d}{d x} \pi(\eta, i, x)=-\bar{\mu}(\eta, x) \pi(\eta, i, x), \\
\pi(\eta, i, 0)=1_{\{i=0\}} \sum_{\zeta \in \mathcal{M}} A(\zeta, \eta) \pi(\zeta) .
\end{gathered}
$$

Therefore, in this case, for $i \neq 0, \pi(\eta, i, x)=0$ and

$$
\bar{\pi}(\eta, x)=\pi(\eta, 0, x)=e^{-\int{ }_{0}^{x} \bar{\mu}(\eta, u) d u} \sum_{\zeta \in \mathcal{M}} A(\zeta, \eta) \pi(\zeta) .
$$

Proposition 5: Let us suppose that the functions $x \rightarrow \pi(\eta, \ell(\xi), x)$ have continuous 
derivatives. Equation $\Pi L f=0$ is satisfied for any $\eta \in \mathcal{H}$, any $i \in \mathcal{L}$ and any function $f \in T(\eta, i)$ if and only if for any $\eta \in \mathcal{M}$ and $\xi \in \mathcal{M}_{\mathrm{g}}$ :

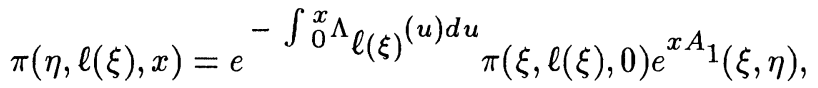

$$
\begin{aligned}
& \pi(\xi, \ell(\xi), 0)=\sum_{\zeta \in \mathscr{P} \cup \mathcal{\&}} \int_{0}^{+\infty} \mu(\zeta, \xi, x) \bar{\pi}(\zeta, x) d x+\sum_{j \in \mathcal{L}} \int_{0}^{+\infty} \Lambda_{j}(x) \pi(\xi, j, x) d x .
\end{aligned}
$$

Proof: Lemma 2 shows that the functions $x \rightarrow \pi(\eta, i, x)(\eta \in \mathcal{M}, i \in \mathcal{L})$ are solutions of the following differential system

Let

$$
\frac{d}{d x} \pi(\eta, i, x)=-\left(q(\eta)+\Lambda_{i}(x)\right) \pi(\eta, i, x)+\sum_{\substack{\zeta \in \mathcal{M} \\ \zeta \neq \eta}} A(\zeta, \eta) \pi(\zeta, i, x) .
$$

$$
\pi_{1}(\eta, i, x)=e^{\int{ }_{0}^{x} \Lambda_{i}(u) d u} \pi(\eta, i, x)
$$

We obtain:

$$
\begin{aligned}
\frac{d}{d x} \pi_{1}(\eta, i, x)= & -q(\eta) \pi_{1}(\eta, i, x)+\sum_{\substack{\zeta \in \mathcal{M} \\
\zeta \neq \eta}} A(\zeta, \eta) \pi_{1}(\zeta, i, x) \\
& =\sum_{\zeta \in \mathcal{M}} A(\zeta, \eta) \pi_{1}(\zeta, i, x)
\end{aligned}
$$

that is to say:

$$
\frac{d}{d x} \pi_{1}(\cdot, i, x)=A_{1}^{T} \pi_{1}(\cdot, i, x)
$$

the matrix $A_{1}^{T}$ being the transpose of matrix $A_{1}$. We deduce that:

and therefore

$$
\pi_{1}(\eta, i, x)=\sum_{\zeta \in \mathcal{H}} \pi(\zeta, i, 0) e^{x A_{1}}(\zeta, \eta)
$$

$$
\pi(\eta, i, x)=e^{-\int{ }_{0}^{x} \Lambda_{i}(u) d u} \sum_{\zeta \in \mathcal{M}} \pi(\zeta, i, 0) e^{x A_{1}}(\zeta, \eta)
$$

The fact that $\pi(\xi, i, 0)=0$ if $\xi \notin \mathcal{H}_{\mathrm{g}}$ or if $i \neq \ell(\xi)$ and the fact that $\ell$ is injective proves the statement.

We must now determine the initial conditions.

Let us denote

- $\quad$ for $\xi \in \mathscr{P} \cup \&, \eta \in \mathcal{M}_{\mathrm{g}}$,

$$
U(\xi, \eta)=\int_{0}^{+\infty} \mu(\xi, \eta, x) e^{-\int_{0}^{x} \bar{\mu}(\xi, u) d u} d x
$$


- for $\xi \in \mathcal{M}_{g}, \eta \in \mathcal{H}$,

$$
\begin{gathered}
V(\xi, \eta)=\int_{0}^{+\infty} \Lambda_{\ell(\xi)}(x) e^{-\int_{0}^{x} \Lambda_{\ell(\xi)}(u) d u} e^{x A_{1}(\xi, \eta) d x} \\
W(\xi, \eta)=\int_{0}^{+\infty} e^{-\int_{0}^{x} \Lambda_{\ell(\xi)}(u) d u} e^{x A_{1}(\xi, \eta) d x}
\end{gathered}
$$

- for $\xi \in \mathcal{M}_{\mathrm{g}}, \eta \in \mathcal{M}_{\mathrm{g}}$

$$
Q(\xi, \eta)=\sum_{\zeta \in \mathscr{S}} W A_{12}(\xi, \zeta) U(\zeta, \eta)+\sum_{\zeta \in \mathcal{M}_{\mathcal{B}}} V(\xi, \zeta) U(\varphi(\zeta), \eta)+V(\xi, \eta)
$$

Proposition 6: Let us suppose that the functions $x \rightarrow \pi(\eta, i, x)$ have continuous derivatives and that Equation $\Pi L f=0$ is satisfied for any $\eta \in \mathbb{E}_{1}, i \in \mathcal{L}$ and $f \in T(\eta, i)$. Then for any $\eta \in \mathcal{M}_{\mathrm{g}}$ :

Proof: Proposition 5 gives

$$
\pi(\eta, \ell(\eta), 0)=\sum_{\xi \in \mathcal{M}_{\mathrm{g}}} \pi(\xi, \ell(\xi), 0) Q(\xi, \eta)
$$

$$
\pi(\eta, \ell(\eta), 0)=\sum_{\zeta \in \mathscr{\Phi} \cup \mathcal{8}} \int_{0}^{+\infty} \mu(\zeta, \eta, x) \bar{\pi}(\zeta, x) d x+\sum_{\zeta \in \mathcal{M}_{\mathrm{g}}} \int_{0}^{+\infty} \Lambda_{\ell(\zeta)}(x) \pi(\eta, \ell(\zeta), x) d x
$$

and

$$
\begin{gathered}
\int_{0}^{+\infty} \Lambda_{\ell(\zeta)}(x) \pi(\eta, \ell(\zeta), x) d x \\
=\int_{0}^{+\infty} \Lambda_{\ell(\zeta)}(x) e^{-\int_{0}^{x} \Lambda_{\ell(\zeta)}(u) d u} e^{x A_{1}(\zeta, \eta) \pi(\zeta, \ell(\zeta), 0) d x} \\
=\pi(\zeta, \ell(\zeta), 0) V(\zeta, \eta) .
\end{gathered}
$$

It gives also, for $\xi \in \mathcal{M}$,

$$
\begin{gathered}
\pi(\xi)=\sum_{\xi^{\prime} \in \mathcal{M}_{\mathrm{g}}} \int_{0}^{+\infty} \pi\left(\xi, \ell\left(\xi^{\prime}\right), x\right) d x \\
=\sum_{\xi^{\prime} \in \mathcal{M}_{\mathrm{g}}} \int_{0}^{+\infty} e^{-\int_{0}^{x} \Lambda_{\ell}\left(\xi^{\prime}\right)^{(u) d u}} \pi\left(\xi^{\prime}, \ell\left(\xi^{\prime}\right), 0\right) e^{x A_{1}\left(\xi^{\prime}, \xi\right) d x} \\
=\sum_{\xi^{\prime} \in \mathcal{M}_{\mathrm{g}}} \pi\left(\xi^{\prime}, \ell\left(\xi^{\prime}\right), 0\right) W\left(\xi^{\prime}, \xi\right) .
\end{gathered}
$$


From Lemma 4, we deduce, for $\zeta \in \mathscr{P}$,

$$
\begin{gathered}
\bar{\pi}(\zeta, x)=e^{-\int_{0}^{x} \bar{\mu}(\zeta, u) d u} \sum_{\xi \in \mathcal{M}} A(\xi, \zeta) \pi(\xi) \\
=e^{-\int_{0}^{x} \bar{\mu}(\zeta, u) d u} \sum_{\xi \in \mathcal{M}} A(\xi, \zeta) \sum_{\xi^{\prime} \in \mathcal{M}_{g}} \pi\left(\xi^{\prime}, \ell\left(\xi^{\prime}\right), 0\right) W\left(\xi^{\prime}, \xi\right) \\
=e^{-\int_{0}^{x} \bar{\mu}(\zeta, u) d u} \sum_{\xi^{\prime} \in \mathcal{M}_{g}} \pi\left(\xi^{\prime}, \ell\left(\xi^{\prime}\right), 0\right) W A_{12}\left(\xi^{\prime}, \zeta\right) .
\end{gathered}
$$

Consequently:

$$
\begin{gathered}
\sum_{\zeta \in \mathscr{\Phi}} \int_{0}^{+\infty} \mu(\zeta, \eta, x) \bar{\pi}(\zeta, x) d x \\
=\sum_{\xi \in \mathcal{M}_{g}} \pi(\xi, \ell(\xi), 0) \sum_{\zeta \in \mathscr{\rho}} \int_{0}^{+\infty} \mu(\zeta, \eta, x) e^{-\int_{0}^{x} \bar{\mu}(\zeta, u) d u} W A_{12}(\xi, \zeta) d x \\
=\sum_{\xi \in \mathcal{M}_{g}} \mu(\xi, \ell(\xi), 0) \sum_{\zeta \in \mathscr{P}} W A_{12}(\xi, \zeta) U(\zeta, \eta) .
\end{gathered}
$$

Similarly, we obtain from Lemma 3 and Proposition 5, for $\zeta \in \mathbb{8}$,

$$
\begin{aligned}
& \bar{\pi}(\zeta, x)=e^{-\int_{0}^{x} \bar{\mu}(\zeta, u) d u} \sum_{\substack{\xi \in \mathcal{M}_{\mathcal{E}} \\
\varphi(\xi)=\zeta}} \sum_{\xi^{\prime} \in \mathcal{M}_{\mathrm{g}}} \int_{0}^{+\infty} \Lambda_{\ell\left(\xi^{\prime}\right)}(x) \pi\left(\xi, \ell\left(\xi^{\prime}\right), x\right) d x \\
& =e^{-\int_{0}^{x} \bar{\mu}(\zeta, u) d u} \sum_{\substack{\xi \in \mathcal{M}_{g} \\
\varphi(\xi)=\zeta}} \sum_{\xi^{\prime} \in \mathcal{M}_{g}} \int_{0}^{+\infty} \Lambda_{\ell\left(\xi^{\prime}\right)}(x) e^{-\int{ }_{0}^{x} \Lambda_{\ell\left(\xi^{\prime}\right)}(u) d u} e^{x A_{1}\left(\xi^{\prime}, \xi\right) d x} \\
& \times \pi\left(\xi^{\prime}, \ell\left(\xi^{\prime}\right), 0\right) \\
& =e^{-\iint_{0}^{x} \bar{\mu}(\zeta, u) d u} \sum_{\xi^{\prime} \in \mathcal{M}_{g}} \pi\left(\xi^{\prime}, \ell\left(\xi^{\prime}\right), 0\right) \sum_{\xi \in \mathcal{M}_{\mathcal{g}}} V\left(\xi^{\prime}, \zeta\right) . \\
& \sum_{\zeta \in \mathcal{E}} \int_{0}^{+\infty} \mu(\zeta, \eta, x) \bar{\pi}(\zeta, x) d x \\
& =\sum_{\xi^{\prime} \in \mathcal{M}_{\mathrm{g}}} \pi\left(\xi^{\prime}, \ell\left(\xi^{\prime}\right), 0\right) \sum_{\zeta \in \mathbb{E}} \sum_{\substack{\xi \in \mathcal{M}_{\mathbb{E}} \\
\varphi(\xi)=\zeta}} V\left(\xi^{\prime}, \xi\right) \int_{0}^{+\infty} \mu(\zeta, \eta, x) e^{-\int_{0}^{x_{\overparen{\mu}}(\zeta, u) d u}} d x
\end{aligned}
$$

Therefore, 


$$
=\sum_{\xi^{\prime} \in \mathcal{M}_{\mathrm{g}}} \pi\left(\xi^{\prime}, \ell\left(\xi^{\prime}\right), 0\right) \sum_{\xi \in \mathcal{M}_{\mathrm{g}}} V\left(\xi^{\prime}, \xi\right) U(\varphi(\xi), \eta) .
$$

So the statement is proved.

Let us call $\mathcal{G}_{1}$ the graph on $\mathcal{H}$ induced by $A_{1}$, i.e., the graph $\mathcal{G}_{1}$ possesses an arrow from $\xi \in \mathcal{H}$ to $\eta \in \mathcal{M}$ iff $A_{1}(\xi, \eta)>0$.

Let us define the following graph $\varrho_{g}$ on $\mathcal{M}_{\mathrm{g}}$ by putting an arrow from $\xi \in \mathcal{H}_{\mathrm{g}}$ to $\eta \in \mathcal{M}_{\mathrm{g}}$ iff one of the following three conditions is satisfied:

(1) there exists a path from $\xi$ to $\eta$ in the graph $g_{1}$;

(2) there exists $\xi^{\prime} \in \mathcal{M}$ and $\zeta \in \mathscr{P}$ such that

- there exists a path from $\xi$ to $\xi^{\prime}$ in the graph $\mathcal{G}_{1}$,

$-A\left(\xi^{\prime}, \zeta\right)=A_{12}\left(\xi^{\prime}, \zeta\right)>0$

- the Lebesgue measure of $(x: \mu(\zeta, \eta, x)>0\}$ is positive;

(3) there exists $\zeta \in \mathcal{M}_{\mathscr{g}}$ such that

- there exists a path from $\xi$ to $\zeta$ in the graph $g_{1}$,

- the Lebesgue measure of $\{x: \mu(\varphi(\zeta), \eta, x)>0\}$ is positive.

Lemma 7: The matrix $Q$ is Markovian. Moreover, if the graph $\mathrm{g}_{\mathrm{g}}$ is irreducible, then the matrix $Q$ is irreducible.

Proof: The entries of the matrixes $A_{12}, U, V, W$ are non-negative, so $Q(\xi, \eta) \geq 0$ for any $\xi$ and $\eta$ in $\mathcal{M}_{\mathrm{g}}$.

On the other hand, for $\zeta \in \mathcal{M}_{\mathrm{g}}$,

$$
\sum_{\eta \in \mathcal{M}_{g}} U(\zeta, \eta)=\int_{0}^{+\infty} \bar{\mu}(\zeta, x) e^{-\int_{0}^{x} \bar{\mu}(\zeta, u) d u} d x=1
$$

Therefore, for $\xi \in \mathcal{M}_{\mathrm{g}}$,

Moreover,

$$
\sum_{\eta \in \mathcal{M}_{g}} Q(\xi, \eta)=\sum_{\zeta \in \mathscr{P}} W A_{12}(\xi, \zeta)+\sum_{\zeta \in \mathcal{M}} V(\xi, \zeta)
$$

$$
\begin{gathered}
\sum_{\zeta \in \mathscr{P}} W A_{12}(\xi, \zeta)=\sum_{\zeta \in \mathscr{P}} \sum_{\eta \in \mathcal{H}} W(\xi, \eta) A(\eta, \zeta) \\
=-\sum_{\eta \in \mathcal{H}} W(\xi, \eta) \sum_{\zeta \in \mathcal{H}} A(\eta, \zeta) \\
=-\sum_{\zeta \in \mathcal{H}} W A_{1}(\xi, \zeta) .
\end{gathered}
$$

Integrating by parts the integral defining $V$, we obtain:

so

$$
V(\xi, \eta)=1_{\{\eta=\xi\}}+\left(W A_{1}\right)(\xi, \eta),
$$

$$
\sum_{\zeta \in \mathscr{P}} W A_{12}(\xi, \zeta)=\sum_{\zeta \in \mathcal{M}}\left(1_{\{\xi=\zeta\}}-V(\xi, \zeta)\right)=1-\sum_{\zeta \in \mathcal{M}} V(\xi, \zeta)
$$


We conclude that

$$
\sum_{\eta \in \mathcal{M}_{g}} Q(\xi, \eta)=1
$$

For proving the irreducibility of $Q$ from the irreducibility of $g_{g}$, we notice that the existence of an arrow from $\xi$ to $\eta$ in the graph $\varrho_{g}$ implies $Q(\xi, \eta)>0$. Indeed if the arrow from $\xi$ to $\eta$ in the graph $g_{g}$ is due to

- $\quad$ condition (1) then, for any $x, e^{x A_{1}}(\xi, \eta)>0$ and therefore $V(\xi, \eta)>0$,

- condition (2) then there exists $\xi^{\prime} \in \mathcal{N}$ such that, for any $x, e^{x A_{1}}\left(\xi, \xi^{\prime}\right)>0$ and therefore $W\left(\xi, \xi^{\prime}\right)>0$, there exists $\zeta \in \mathscr{P}$ such that $A_{12}\left(\xi^{\prime}, \zeta\right)>0$ and $U(\zeta, \eta)>0$. So

$$
\begin{gathered}
\sum_{\zeta_{1} \in \mathscr{P}} W A_{12}\left(\xi, \zeta_{1}\right) U\left(\zeta_{1}, \eta\right)=\sum_{\zeta_{1} \in \mathscr{\rho}} \sum_{\xi_{1}^{\prime} \in \mathcal{M}} W\left(\xi, \xi_{1}^{\prime}\right) A_{12}\left(\xi_{1}^{\prime}, \zeta_{1}\right) U\left(\zeta_{1}, \eta\right), \\
\geq W\left(\xi, \xi^{\prime}\right) A_{12}\left(\xi^{\prime}, \zeta\right) U(\zeta, \eta)>0 .
\end{gathered}
$$

- condition (3) then there exists $\zeta \in \mathcal{M}_{\mathcal{g}}$ such that $V(\xi, \zeta)>0$ and $U(\varphi(\zeta), \eta)>0$, therefore

$$
\sum_{\zeta_{1} \in \mathcal{M}_{\mathbb{8}}} V\left(\xi, \zeta_{1}\right) U\left(\varphi\left(\zeta_{1}\right), \eta\right) \geq V(\xi, \zeta) U(\varphi(\zeta), \eta)>0
$$

Proposition 8: Let us suppose that the graph $\mathrm{G}_{\mathrm{g}}$ is irreducible. Then the system of equations

$$
z(\eta)=\sum_{\xi \in \mathcal{M}_{\mathrm{g}}} z(\xi) Q(\xi, \eta), \quad \eta \in \mathcal{M}_{\mathrm{g}}
$$

has a solution $z_{0}$ such that $z_{0}(\eta) \geq 0$ for all $\eta \in \mathcal{M}_{\mathrm{g}}$, and $\sum_{\eta \in \mathcal{M}_{\mathrm{g}}} z_{0}(\eta)>0$. Moreover, any solution $z$ of this system satisfies $z(\eta)=c z_{0}(\eta)$ where $c$ is a constant.

Proof: The matrix $Q$ is aperiodic since, for any $\xi \in \mathcal{M}_{g}$ and any $x \in \mathbb{R}_{+}$, $e^{x A_{1}}(\xi, \xi)>0$ and therefore $Q(\xi, \xi) \geq V(\xi, \xi)>0$. Then the proposition is an application of the Perron-Frobenius Theorem.

The following proposition resumes the formulas obtained by writing them in matrix form as far as possible.

Proposition 9: Let us suppose that the graph $\mathrm{g}_{\mathrm{g}}$ is irreducible and that the stationary measure $\Pi$ has a density $\pi$ such that the functions $x \rightarrow \pi(\eta, i, x)$ have continuus derivatives. Let $z_{0}$ be a nontrivial solution of the system

$$
z(\eta)=\sum_{\xi \in \mathcal{M}_{\mathrm{g}}} z(\xi) Q(\xi, \eta), \quad \eta \in \mathcal{M}_{\mathrm{g}}
$$

Then, for any $\eta \in \mathcal{M}$ and $\xi \in \mathcal{M}_{\mathrm{g}}$ :

$$
\pi(\eta, \ell(\xi), x)=c z_{0}(\xi) e^{-\int_{0}^{x} \Lambda_{\ell(\xi)}(u) d u} e^{x A_{1}}(\xi, \eta)
$$


and therefore for any $\eta \in \mathcal{M}$ :

For $\eta \in \mathfrak{P}:$

$$
\begin{gathered}
\pi(\eta)=c\left(z_{0} W\right)(\eta) . \\
\pi(\eta, i, x)=0 \text { if } i \neq 0 \\
\bar{\pi}(\eta, x)=\pi(\eta, 0, x)=e^{-\int_{0}^{x} \bar{\mu}(\eta, u) d u} \sum_{\xi \in \mathcal{M}} A(\xi, \eta) \pi(\xi),
\end{gathered}
$$

consequently, if $m(\eta)$ is the mean sojourn time in the state $\eta \in \mathscr{P}$ :

$$
\pi(\eta)=m(\eta) \sum_{\xi \in \mathcal{M}} A(\xi, \eta) \pi(\xi)=c m(\eta)\left(z_{0} W A_{12}\right)(\eta)
$$

For $\eta \in$ \&:

and therefore:

$$
\begin{gathered}
\pi(\eta, i, x)=0 \text { if } i \neq 0, \\
\bar{\pi}(\eta, x)=\pi(\eta, 0, x)=c e^{-\int_{0}^{x} \bar{\mu}(\eta, u) d u} \sum_{\substack{\xi \in \mathcal{M}_{\mathcal{E}} \\
\varphi(\xi)=\eta}}\left(z_{0} V\right)(\xi),
\end{gathered}
$$

$$
\pi(\eta)=c m(\eta) \sum_{\substack{\xi \in \mathcal{M}_{\&} \\ \varphi(\xi)=\eta}}\left(z_{0} V\right)(\xi)
$$

$m(\eta)$ being the mean sojourn time in the state $\eta \in \mathbb{8}$.

Proof: This proposition is directly obtained from Lemma 3 and 4 and Propositions 5, 6 and 8 . Let us note that, for $\eta \in \mathscr{P} \cup \mathcal{E}, \sum_{\xi \in \mathcal{M}_{g}} \mu(\eta, \xi, x)=\bar{\mu}(\eta, x)$ is the hazard rate of
the duration in the state $\eta$. Therefore,

$$
m(\eta)=\int_{0}^{+\infty} e^{-\int_{0}^{x} \bar{\mu}(\eta, u) d u} d x
$$

is the mean sojourn time in the state $\eta$.

Remark 10: Proposition 9 shows that:

$$
\begin{gathered}
\forall \eta \in \mathscr{P}, \sum_{\xi \in \mathcal{H}_{0}} A(\xi, \eta) \pi(\xi)=\frac{\pi(\eta)}{m(\eta)}, \\
\forall \eta \in \mathcal{\&}, \sum_{\substack{\xi \in \mathcal{H}_{\mathbb{8}} \\
\varphi(\xi)=\eta}} c\left(z_{0} V\right)(\xi)=\frac{\pi(\eta)}{m(\eta)} .
\end{gathered}
$$

Theorem 11: The measure $\Pi=(\pi(\eta, i, x))$ described in Proposition 9 is a stationary measure.

Proof: It suffices to prove that measure $\Pi$ given in Proposition 9 verifies the condition $\Pi L f=0$ for any $\eta \in E_{1}, i \in \mathcal{L}$ and any function $f \in T(\eta, i)$. Lemmas 2,3 
and 4 show that it suffices to verify that

- for $\eta \in \mathcal{H}, \xi \in \mathcal{M}_{\mathrm{g}}$,

$$
\frac{d}{d x} \pi(\eta, \ell(\xi), x)=-\left(q(\eta)+\Lambda_{\ell(\xi)}(x)\right) \pi(\eta, \ell(\xi), x)+\sum_{\substack{\zeta \in \mathcal{M} \\ \zeta \neq \eta}} A(\zeta, \eta) \pi(\zeta, \ell(\xi), x),
$$

$$
\begin{aligned}
& \pi(\eta, \ell(\xi), 0)=1_{\{\eta=\xi\}} \\
& \quad \times\left(\sum_{\zeta \in \mathcal{P}_{\mathcal{B}}} \int_{0}^{+\infty} \mu(\zeta, \eta, x) \bar{\pi}(\zeta, x) d x+\sum_{\zeta \in \mathcal{M}_{\mathrm{g}}} \int_{0}^{+\infty} \Lambda_{\ell(\zeta)}(x) \pi(\eta, \ell(\zeta), x) d x\right),
\end{aligned}
$$

- $\quad$ for $\eta \in \mathcal{E}, i \in \mathcal{L}$,

$$
\begin{gathered}
\frac{d}{d x} \pi(\eta, i, x)=-\bar{\mu}(\eta, x) \pi(\eta, i, x) \\
\pi(\eta, i, 0)=1_{\{i=0\}} \sum_{\substack{\zeta \in \mathcal{M}_{\mathcal{E}} \\
\varphi(\zeta)=\eta}} \sum_{\xi \in \mathcal{M}_{g}} \int_{0}^{+\infty} \Lambda_{\ell(\xi)}(x) \pi(\zeta, \ell(\xi), x) d x
\end{gathered}
$$

- $\quad$ for $\eta \in \mathscr{P}, i \in \mathcal{L}$,

$$
\begin{gathered}
\frac{d}{d x} \pi(\eta, i, x)=-\bar{\mu}(\eta, x) \pi(\eta, i, x), \\
\pi(\eta, i, 0)=1_{\{i=0\}} \sum_{\zeta \in \mathcal{M}} A(\zeta, \eta) \pi(\zeta) .
\end{gathered}
$$

This verification is easily done using $\frac{d}{d x} e^{x A_{1}}=e^{x A_{1}} A_{1}$, and $z_{0} Q=z_{0}$.

\section{Calculating Average Cost}

We suppose that the following are known:

- $\quad C_{\mathrm{g}}$ : the cost of an inspection (displacement cost),

- $\quad C_{P \&}(\eta)$ : the cost of replacement pieces for maintenance corresponding to the state $\eta \in \mathbb{8}$,

- $\quad C_{H \&}(\eta)$ : the hourly labor cost for maintenance corresponding to the state $\eta \in \mathbb{8}$,

- $\quad C_{I P}$ : the hourly cost of the predicted immobilization of material (that which is due to the maintenance),

- $\quad C_{\Phi \rho}(\eta)$ : the cost of replacement pieces for repair corresponding to the failure state $\eta \in \mathscr{P}$, including displacement costs,

- $\quad C_{H \mathscr{P}}(\eta)$ : the hourly cost of labor for the repair corresponding to the failure state $\eta \in \mathscr{\rho}$,

- $\quad C_{I N P}:$ the hourly cost of an unpredicted immobilization of the material (due to the repair).

Now let us note $N_{\mathrm{g}}(t)$ the number of inspections carried out in the time interval 
$[0, t], N_{\eta, \xi}(t)$ the number of jumps of the process $\left(\Phi_{s}\right)$ from $\eta$ to $\xi$ during the time interval $[0, t]$ and $C(t)$ the cost of using the material during this period.

It is clear that

$$
\begin{aligned}
& C(t)=C_{g} N_{g}(t)+\sum_{\xi \in \mathcal{E}} C_{P \mathscr{E}}(\xi) \sum_{\eta \in \mathcal{M}_{\mathcal{E}}} N_{\eta, \xi}(t)+\sum_{\eta \in \mathcal{E}} C_{H \mathcal{E}}(\eta) \int_{0}^{t} 1_{\left\{\Phi_{u}=\eta\right\}} d u \\
& +C_{I P} \int_{0}^{t} 1_{\left\{\Phi_{u} \in \mathcal{E}\right\}} d u+\sum_{\xi \in \mathscr{\mathcal { P }}} C_{\mathscr{\rho}}(\xi) \sum_{\eta \in \mathcal{M}} N_{\eta, \xi}(t) \\
& +\sum_{\eta \in \mathscr{\Phi}} C_{H \mathscr{P}}(\eta) \int_{0}^{t} 1_{\left\{\Phi_{u}=\eta\right\}} d u+C_{I N P} \int_{0}^{t} 1_{\left\{\Phi_{u} \in \mathscr{P}\right\}} d u \text {. }
\end{aligned}
$$

The asymptotic average cost required is:

$$
C=\lim _{t \rightarrow+\infty} \frac{1}{t} \mathbb{E}(C(t))
$$

General results for semi-regenerative processes ([1]) prove that, if $\xi$ is a regeneration point of the process $\left(\Phi_{t}\right)$ then the average number of visits to $\xi$ before $t$, denoted $n v_{\xi}(t)$ satisfies

$$
\lim _{t \rightarrow \infty} \frac{n v_{\xi}(t)}{t}=\lim _{t \rightarrow \infty} \frac{\mathbb{E}\left(\sum_{\eta} N_{\eta, \xi}(t)\right)}{t}=\frac{\pi(\eta)}{m(\eta)}
$$

where $m(\eta)$ is the mean sojourn time in the state $\eta$. This result can be applied in our model for $\eta \in \mathscr{P} \cup \&$.

Below, we are going to rediscover this result using martingale techniques. To calculate the average number of jumps from $\eta$ to $\xi$, we apply the following lemma:

Lemma 12:

where

$$
\frac{1}{t} \mathbb{E}\left(N_{\eta, \xi}(t)\right) \underset{t \rightarrow+\infty}{\longrightarrow} \sum_{i} \int_{0}^{+\infty}\left(L f_{\xi}\right)(\eta, i, x) \pi(\eta, i, x) d x
$$

$$
f_{\xi}(\zeta, i, x)=1_{\{\zeta=\xi\}}
$$

Proof: The classic results on Markov process show that for any $\xi \in E_{1}$, the process $M_{\xi}$, defined by

$$
M_{\xi}(t)=1_{\left\{\Phi_{t}=\xi\right\}}-1_{\left\{\Phi_{0}=\xi\right\}}-\int_{0}^{t}\left(L f_{\xi}\right)\left(\Phi_{u}, I_{u}, X_{u}\right) d u
$$

is a martingale. It is therefore the same for

$$
\int_{0}^{t} 1_{\left\{\Phi_{u-}=\eta\right\}} d M_{\xi}(u)=N_{\eta, \xi}(t)-\int_{0}^{t} 1_{\left\{\Phi_{u}=\eta\right\}}\left(L f_{\xi}\right)\left(\eta, I_{u}, X_{u}\right) d u .
$$

By taking the expectation, we obtain 


$$
\mathbb{E}\left(N_{\eta, \xi}(t)\right)=\int_{0}^{t} \mathbb{E}\left(1_{\left\{\Phi_{u}=\eta\right\}}\left(L f_{\xi}\right)\left(\eta, I_{u}, X_{u}\right)\right) d u
$$

The distribution of $\left(\Phi_{u}, I_{u}, X_{u}\right)$ converging towards $\Pi$, the result comes from here.

We can deduce the following results without difficulty:

- $\quad$ for $\eta \in \mathcal{M}, \xi \in E, \eta \neq \xi$,

$$
\frac{1}{t} \mathbb{E}\left(N_{\eta, \xi}(t)\right) \underset{t \rightarrow \infty}{\longrightarrow} A(\eta, \xi) \pi(\eta),
$$

- $\quad$ for $\eta \in \mathscr{P} \cup \&, \xi \in E, \eta \neq \xi$,

$$
\frac{1}{t} \mathbb{E}\left(N_{\eta, \xi}(t)\right) \underset{t \rightarrow \infty}{\longrightarrow} 1_{\left\{\xi \in \mathcal{M}_{g}\right\}} U(\eta, \xi) \frac{\pi(\eta)}{m(\eta)},
$$

- for $\eta \in E, \xi \in \&$,

$$
\begin{gathered}
\left.\frac{1}{t} \mathbb{E}\left(N_{\eta, \xi}(t)\right) \underset{t \rightarrow+\infty}{\longrightarrow} 1 \eta \in \mathcal{M}_{\mathcal{Q}}, \varphi(\eta)=\xi\right\} \sum_{\zeta \in \mathcal{M}_{g}} \int_{0}^{+\infty} \Lambda_{\ell(\zeta)}(x) \pi(\eta, \ell(\zeta), x) d x \\
=1_{\left\{\eta \in \mathcal{M}_{\mathcal{g}}, \varphi(\eta)=\xi\right\}} c\left(z_{0} V\right)(\eta) .
\end{gathered}
$$

Remark 13: The first result and Remark 10 show that, for $\xi \in \mathscr{P}$,

$$
\frac{1}{t} \sum_{\eta \in \mathcal{M}} \mathbb{E}\left(N_{\eta, \xi}(t)\right) \underset{t \rightarrow \infty}{\longrightarrow} \sum_{\eta \in \mathcal{M}} A(\eta, \xi) \pi(\eta)=\frac{\pi(\xi)}{m(\xi)}
$$

The third result above and Remark 10 show that, for $\xi \in \mathbb{8}$,

$$
\frac{1}{t} \sum_{\eta \in E} \mathbb{E}\left(N_{\eta, \xi}(t)\right) \underset{\substack{t \rightarrow \infty \\ \eta \in \mathcal{M}_{\mathcal{E}} \\ \varphi(\eta)=\xi}}{\longrightarrow} c\left(z_{0} V\right)(\eta)=\frac{\pi(\xi)}{m(\xi)} .
$$

These results have been announced above.

However, these results do not allow us to calculate the average number of inspections. To do this, we must add into the process a supplementary variable which counts the number of inspections. The generator of the process completed in this way is written:

$$
\begin{aligned}
(L f)(\zeta, j, x, n)= & 1_{\left\{\zeta \in \mathcal{M}_{\}}\right\}} \sum_{\substack{\xi \in \mathcal{M} \\
\xi \neq \zeta}} A(\zeta, \xi)[f(\xi, j, x, n)-f(\zeta, j, x, n)] \\
& +1_{\left\{\zeta \in \mathcal{M}_{\}}\right\}} \sum_{\xi \in \mathscr{\mathcal { O }}} A(\zeta, \xi)[f(\xi, 0,0, n)-f(\zeta, j, x, n)] \\
& +1_{\{\zeta \in \mathscr{P} \cup \&\}} \sum_{\xi \in \mathcal{M}_{g}} \mu(\zeta, \xi, x)[f(\xi, \ell(\xi), 0, n)-f(\zeta, j, x, n)] \\
& +1_{\left\{\zeta \in \mathcal{M}_{g}\right\}} \Lambda_{j}(x)[f(\zeta, \ell(\zeta), 0, n+1)-f(\zeta, j, x, n)] \\
& +1_{\left\{\eta \in \mathcal{M}_{g}\right\}} \Lambda_{j}(x)[f(\varphi(\zeta), 0,0, n+1)-f(\zeta, j, x, n)]
\end{aligned}
$$




$$
+\frac{\partial f}{\partial x}(\zeta, j, x, n)
$$

By using the martingale method described earlier (proof of Lemma 12), we obtain

$$
\frac{1}{t} \mathbb{E}\left(N_{\mathrm{g}}(t)\right) \underset{t \rightarrow+\infty}{\longrightarrow} \sum_{\eta \in \mathcal{M}} \sum_{\xi \in \mathcal{M}_{\mathrm{g}}} \int^{+\infty} \Lambda_{\ell(\xi)}(x) \pi(\eta, \ell(\xi), x) d x=\sum_{\eta \in \mathcal{N}} c\left(z_{0} V\right)(\eta) .
$$

The above results and Remark 10 give:

Theorem 14: The average operating cost over the infinite horizon is:

$$
\begin{gathered}
C=\lim _{\substack{t \rightarrow+\infty \\
t}} \frac{1}{\mathbb{E}} \mathbb{E}(C(t) \\
=c C_{\mathrm{g}} \sum_{\eta \in \mathcal{M}}\left(z_{0} V\right)(\eta)+\sum_{\eta \in \mathcal{E}}\left(\frac{C_{P \mathcal{E}}(\eta)}{m(\eta)}+C_{H \mathcal{E}}(\eta)+C_{I P}\right) \pi(\eta) \\
+\sum_{\eta \in \mathscr{P}}\left(\frac{C_{P}(\eta)}{m(\eta)}+C_{H \mathscr{P}}(\eta)+C_{I N P}\right) \pi(\eta) .
\end{gathered}
$$

Remark 15: We note that in usual cases, this asymptotic cost depends on the average duration of repairs only and not on the forms of the distributions.

Remark 16: By using the ergodic theorem for regenerative processes, we can show that $C(t) / t$ converges almost surely towards $C$.

\section{Numerical Results}

\subsection{Calculating Cost}

We assume that the lengths of time between inspections have a gamma distribution. The parameters of the distribution corresponding to the label $i$ are denoted by $\alpha_{i}$ and $\beta_{i}$; its p.d.f. is therefore

$$
f_{i}(x)=\frac{1}{\Gamma\left(\alpha_{i}\right) \beta_{i}^{\alpha_{i}}} x^{\alpha_{i}-1} e^{-x / \beta_{i}}
$$

Let $F_{i}$ denote the distribution function of $f_{i}$ and let $\bar{F}_{i}=1-F_{i}$.

The only difficulty is to calculate $W$ :

$$
\begin{aligned}
W(i, j) & =\int_{0}^{+\infty} e^{-\int_{0}^{x} \Lambda_{i}(u) d u} e^{x A_{1}}(i, j) d x \\
& =\int_{0}^{+\infty} \bar{F}_{i}(x) e^{x A_{1}(i, j) d x .}
\end{aligned}
$$


We suppose that the $A_{1}$ matrix is diagonizable therefore, we note $P$ a matrix such that matrix $P^{-1} A_{1} P$ is diagonal. Let $(d(r))_{1 \leq r \leq m}$ be the eigenvalues of $A_{1}$. We obtain:

$$
\left(e^{x A_{1}}\right)(i, j)=\sum_{r=1}^{m} P(i, r) e^{x d(r)} P^{-1}(r, j),
$$

and

$$
W(i, j)=\sum_{r=1}^{m} P(i, r) P^{-1}(r, j) \bar{F}_{i}^{*}(-|d(r)|),
$$

where $\bar{F}_{i}^{*}$ is the Laplace transform of $\bar{F}_{i}$. We have

$$
\forall s>0, \quad \bar{F}_{i}^{*}(s)=\frac{1}{s}-\frac{1}{s} \frac{1}{\left(1+\beta_{i} s\right)^{\alpha}},
$$

and the expression for $W(i, j)$ can be deduced immediately.

\subsection{Optimization}

We first choose the initial model and the set $\mathcal{M}_{\mathrm{g}}$ of working states which do not lead to maintenance, i.e., those which are labeled. Let $m_{1}$ be its cardinal. We also suppose that the costs of inspection, maintenance and so on, defined in the beginning of paragraph 3 , are fixed. We will determine the parameters $\left(\alpha_{i}, \beta_{i}\right)_{1 \leq i \leq m_{1}}$ which minimize the cost $C$.

For this, we use a simulated annealing algorithm based on a Metropolis algorithm. We start by resetting the parameters of the gamma distributions using their expectations and their standard deviations, i.e., we define:

$$
m_{i}=\alpha_{i} \beta_{i}, \quad \sigma_{i}=\beta_{i} \sqrt{\alpha_{i}} .
$$

Let us suppose that these values $\left(m_{i}, \sigma_{i}\right)_{1<i<m_{1}}$ are those obtained from the iteration $n-1$ and that the associated cost is $C$. The iteration $n$ of the algorithm is carried out in the following way (according to three real parameters $a, b, q$, which are strictly positive, initially fixed and therefore do not depend on $n$ ):

- choose the $i$ label (for which the distribution is to be changed eventually) with the uniform distribution on $\left\{1, \ldots, m_{1}\right\}$,

- choose the value $m^{\prime}(i)$ following the normal distribution with average $m(i)$ and variance $a^{2}$,

- $\quad$ choose $\sigma_{i}^{\prime}$ following the lognormal distribution described as: the distribution of $\log \sigma_{i}^{\prime}$ is normal with average $\log \sigma_{i}$ and variance $b^{2}$,

- $\quad$ calculate the new cost $C$ by replacing $\left(m_{i}, \sigma_{i}\right)$ with $\left(m_{i}^{\prime}, \sigma_{i}^{\prime}\right)$ without modifying the others $\left(m_{j}, \sigma_{j}\right)$. (The calculation of this new cost means we must return to the initial set of parameters),

- $\quad$ keep the new values $\left(m_{i}^{\prime}, \sigma_{i}^{\prime}\right)$ with probability

$$
\max \left(e^{-\theta_{n}\left(C^{\prime}-C\right)}, 1\right)
$$

where $\theta_{n}=q \log n$.

After that, we can find the best set $\mathcal{M}_{\mathrm{g}}$ by taking successively all different sets and performing the above optimization on each set. 


\subsection{Examples}

\subsubsection{Example 1}

The initial model is a $k$ out of $n$ system. It corresponds to $n$ identical components with a constant failure rate, in active redundancy (warm standby). The system works if and only if at least $k$ components are working. After repairs or services (i.e., corrective or preventive maintenance), the system comes back to the nominal state corresponding to $n$ working components.

We suppose that:

- $\quad k=2$,

- $\lambda=1$,

- the average length of a repair equals $1 / 50$,

- the average length of a maintenance period equals $(i+1) / 1000$, if there are exactly $i$ components broken when the maintenance is carried out,

- the cost of an inspection equals 1 ,

- the cost of replacement pieces for the maintenance corresponding to a state where there are exactly $i$ broken components is equal to $i$,

- the hourly cost for labor for maintenance is equal to 1 ,

- the hourly cost of a predicted immobilization equals 0.5 ,

- the cost of displacement for repair, added to the cost of replacement pieces equals 20 ,

- the hourly labor cost for a repair equals 25 ,

- the hourly cost of an unpredicted immobilization costs 75 .

The set $\mathcal{M}_{\mathrm{g}}$ is formed of states having at most $m_{1}-1$ broken components. The results obtained according to $n$ and $m_{1}$ are in Table 1 .

\begin{tabular}{|l|c|c|c|c|c|c|c|}
\hline $\begin{array}{l}m_{1} \\
n\end{array}$ & 1 & 2 & 3 & 4 & 5 & 6 & 7 \\
\hline 3 & 16.151 & & & & & & \\
\hline 4 & 10.139 & 12.260 & & & & & \\
\hline 5 & 8.458 & 8.871 & 10.693 & & & & \\
\hline 6 & 7.926 & 7.995 & 8.381 & 9.889 & & & \\
\hline 7 & 7.809 & 7.813 & 7.874 & 8.176 & 9.435 & & \\
\hline 8 & 7.864 & 7.863 & 7.872 & 7.901 & 8.126 & 9.170 & \\
\hline 9 & 7.997 & 7.996 & 7.992 & 7.975 & 7.984 & 8.149 & 9.000 \\
\hline
\end{tabular}

Table 1

We see that the minimum cost is obtained with 7 components, and only the nominal working state giving inspection is being unassociated with maintenance. These results are obviously highly dependent on the initial costs imposed. In the chosen example, the costs associated with unpredicted immobilization and repairs (following a system failure) are high compared to the other costs. This explains the fact that risks should not be taken and preventive maintenance should be carried out as soon as an inspection detects a broken component, at the very least when there are not too many broken components ( 7 or less). However, we do not have an intuitive explanation justifying the fact that the minimum cost is obtained with 7 components.

The most remarkable phenomenon that we have observed is that, in each case, the 
minimum is obtained for values of $\sigma_{i}$ almost zero (about $10^{-7}$ or smaller, the influence of the cost when these values are even smaller being null). This shows that the optimal strategy consists of associating a non-random inspection length to each state of $\mathcal{M}_{\mathrm{g}}$. We think that it is a very important result in practice and easy to use, but we do not know how to justify this phenomenon mathematically.

The different optimal lengths found in the different cases are shown in Table 2.

It should be noted that the cost function seems "quite flat" next to its minimum. Different values can give the same minimum cost. For example in the case $n=7$, $m_{1}=5$, the following lengths between inspection give a cost of 9.435 :

$$
\begin{array}{lllll}
0.787 & 0.667 & 0.529 & 0.334 & 0.186
\end{array}
$$

As the $n$ and $m_{1}$ numbers are fixed, the optimization algorithm requires the adjustment of some of the constants. In the example presented, we have taken $a=0.1$ and $b=1$. The setting of the constant $q$ is more difficult. If it is too low, the algorithm takes too long to converge; if it is too high, the algorithm risks being trapped in a local minimum of cost function. The influence of this $q$ constant is shown on the graphs on the following pages. Here the 7 component case is treated, the cardinal of $\mathcal{M g}_{\mathrm{g}}$ being equal to 4 . Respectively, the $q$ constant is taken to be equal to 10 and 10000 , with the initial values of the parameters being identical. The initial values of the parameters correspond to lengths between inspections following exponential distributions with intuitively reasonable averages.

In practice, we started by putting a relatively low value of $q$ (between 10 and 100) through the algorithm using different initial points. This allowed us to quickly determine good approximations of $m_{i}$ and $\sigma_{i}$ giving minimum cost. We then refined the method by using these approximations as initial values and by taking higher values for the $q$ constant (between 10,000 and 100,000).

In fact, it turns out that with a reasonable starting point such as exponential distribution, the average of which is half the MTTF of the initial process (the initial state being that in consideration), and a $q$ value of 10,000 , we obtain, in general, a precise value for the minimum. More precisely, the values obtained for $m_{i}$ and $\sigma_{i}$ vary little between different applications of the algorithm. On the other hand, the values for $\alpha_{i}$ and $\beta_{i}$ are considerably different. This can be explained in the following way: the variances can be considered to be null and the $m_{i}$ values are significant, whereas, in practice, as the values of $\beta_{i}$ (as those of $\sigma_{i}$ ) are very low, their exact value is insignificant, and their fluctuations bring on those of $\alpha_{i}=m_{i} / \beta_{i}$. 


\begin{tabular}{|c|c|c|c|c|c|c|c|}
\hline $\begin{array}{l}m_{1} \\
\mathrm{n}\end{array}$ & 1 & 2 & 3 & 4 & 5 & 6 & 7 \\
\hline 3 & 0.174 & & & & & & \\
\hline 4 & 0.264 & $\begin{array}{l}0.341 \\
0.165\end{array}$ & & & & & \\
\hline 5 & 0.375 & $\begin{array}{l}0.403 \\
0.255\end{array}$ & $\begin{array}{l}0.492 \\
0.340 \\
0.168\end{array}$ & & & & \\
\hline 6 & 0.493 & $\begin{array}{l}0.498 \\
0.374\end{array}$ & $\begin{array}{l}0.540 \\
0.414 \\
0.258\end{array}$ & $\begin{array}{l}0.636 \\
0.500 \\
0.351 \\
0.172\end{array}$ & & & \\
\hline 7 & 0.612 & $\begin{array}{l}0.617 \\
0.502\end{array}$ & $\begin{array}{l}0.634 \\
0.507 \\
0.384\end{array}$ & $\begin{array}{l}0.659 \\
0.555 \\
0.417 \\
0.264\end{array}$ & $\begin{array}{l}0.772 \\
0.638 \\
0.507 \\
0.356 \\
0.178\end{array}$ & & \\
\hline 8 & 0.745 & $\begin{array}{l}0.741 \\
0.648\end{array}$ & $\begin{array}{l}0.727 \\
0.541 \\
0.523\end{array}$ & $\begin{array}{l}0.754 \\
0.495 \\
0.559 \\
0.373\end{array}$ & $\begin{array}{l}0.790 \\
0.724 \\
0.569 \\
0.443 \\
0.262\end{array}$ & $\begin{array}{l}0.899 \\
0.811 \\
0.682 \\
0.522 \\
0.347 \\
0.187\end{array}$ & \\
\hline 9 & 0.878 & $\begin{array}{l}0.878 \\
0.791\end{array}$ & $\begin{array}{l}0.873 \\
0.788 \\
0.688\end{array}$ & $\begin{array}{l}0.873 \\
0.781 \\
0.668 \\
0.548\end{array}$ & $\begin{array}{l}0.867 \\
0.784 \\
0.673 \\
0.543 \\
0.419\end{array}$ & $\begin{array}{l}0.908 \\
0.812 \\
0.707 \\
0.576 \\
0.460 \\
0.286\end{array}$ & $\begin{array}{l}1.036 \\
0.912 \\
0.811 \\
0.688 \\
0.547 \\
0.381 \\
0.195\end{array}$ \\
\hline
\end{tabular}

Table 2 

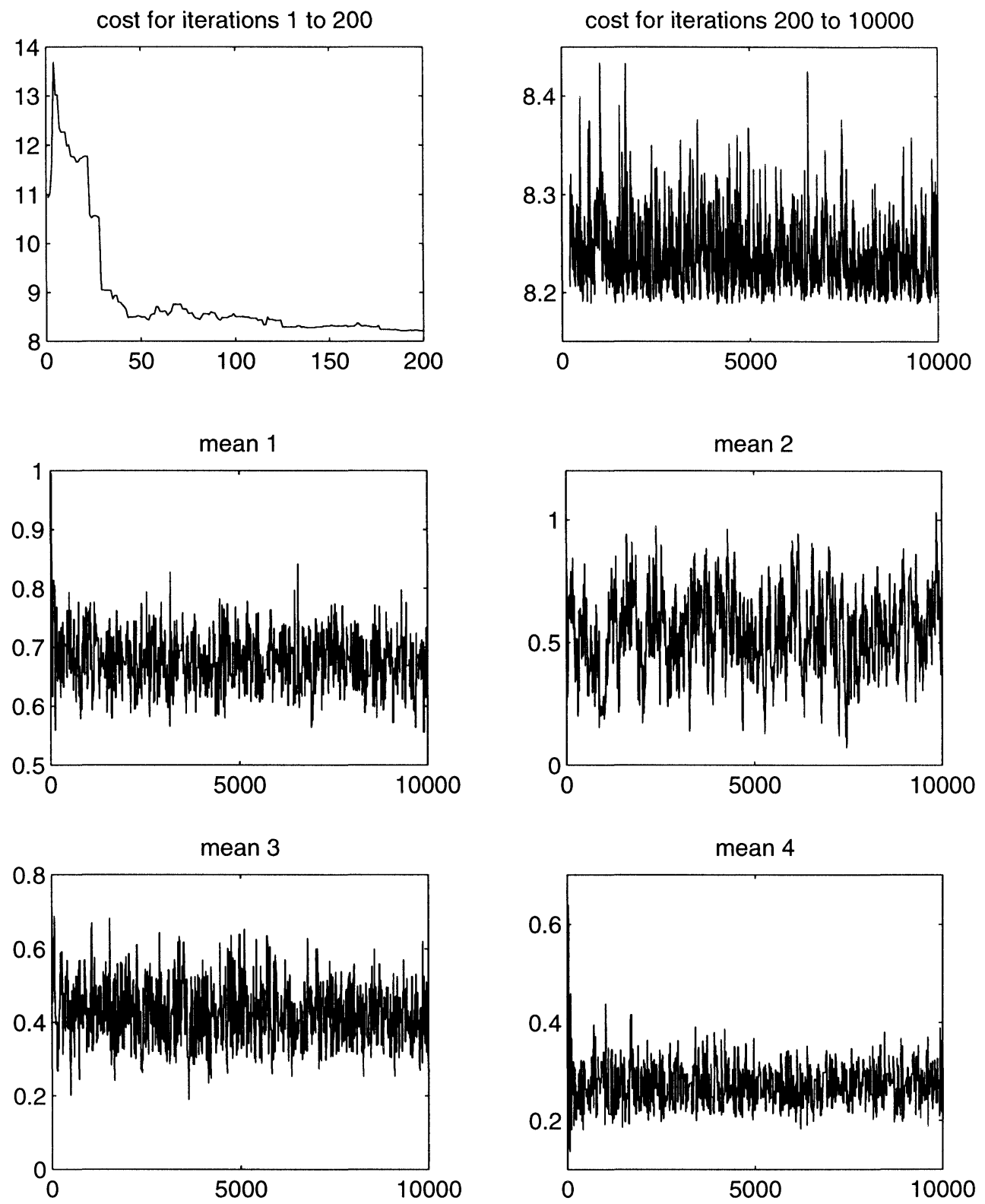

Cost and means versus number of iterations, $q=10$. 

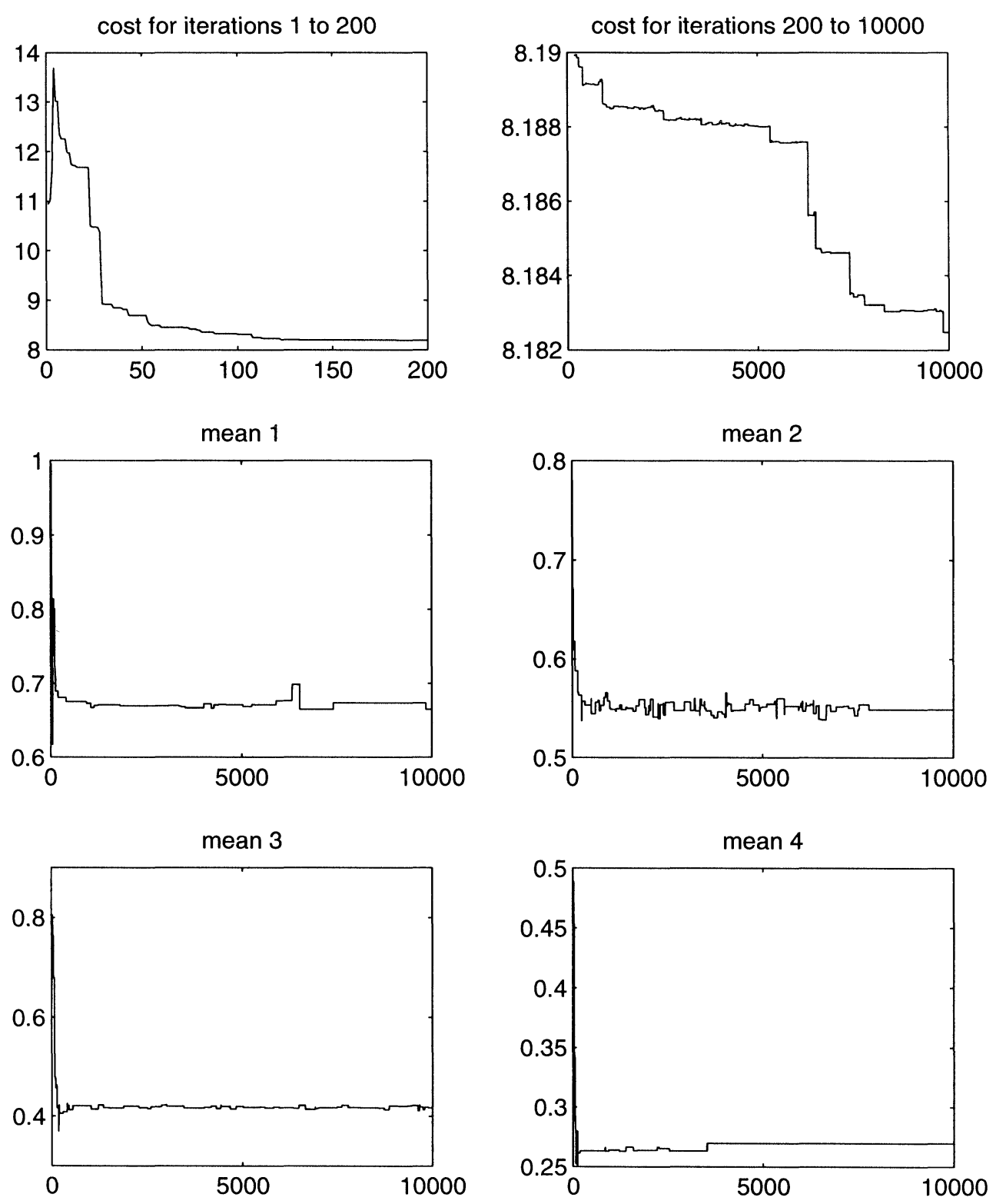

Cost and means versus number of iterations, $q=10,000$. 


\subsubsection{Example 2}

In the first example, the initial process was a birth and death process. This hypothesis is not at all necessary to make the proposed method work, as the second example will show. After corrective or preventive maintenance, the system comes back to the nominal state corresponding to state 1 .

We take into consideration, an initial system with six states: four working states (states 1 to 4 ) and two failure states (states 5 and 6 ). The transition from the working states (meaning the strictly positive terms of the $A$ matrix) are:

$$
\begin{gathered}
A(1,2)=2, \quad A(1,4)=1 \\
A(2,3)=2, \quad A(3,5)=1.5 \\
A(4,5)=0.5, \quad A(4,6)=1 .
\end{gathered}
$$

The average repair lengths are $1 / 25$ for state 5 and $1 / 50$ for state 6 .

The average maintenance lengths associated with states 2,3 and 4 are $1 / 1000$, $2 / 1000$, and $2 / 1000$, respectively. The costs of replacement pieces are 1,3 , and 2 , respectively, and the hourly cost of labor for all is 1 .

The cost of an inspection is 1 , that of a predicted immobilization (due to a maintenance) is 0.5 and that of an unpredicted immobilization (due to a repair) is 75 .

The cost of replacement pieces for repairs plus displacement costs for states 5 and 6 are 20 and 30, respectively. The hourly cost of labor for these repairs is 75 .

The results obtained are summarized in the following table (in each case, the $m_{i}$ values have been presented vertically, index $i$ being in ascending order):

\begin{tabular}{|l|l|l|l|l|l|}
\hline $\mathcal{M}_{\mathrm{g}}$ & \multicolumn{1}{|c|}{1} & $\{1,2\}$ & $\{1,2,3\}$ & $\{1,4\}$ & $\{1,2,4\}$ \\
\hline minimum cost & 11.795 & 12.238 & 17.038 & 17.363 & 16.647 \\
\hline$m_{i}$ & 0.275 & 0.294 & 0.308 & 0.886 & 0.631 \\
values & & 0.251 & 5.432 & 13.925 & 11.397 \\
& & & 4.176 & & 0.305 \\
\hline
\end{tabular}

Once again, the minimum is obtained with a single state of inspection ( $\mathcal{H}_{\mathrm{g}}$ equals $\{1\}$ ), the reasons seemingly being the same as in the first example (high values relating to the various costs associated with repair).

We also find the same phenomenon in regard to the variances as in Example 1. Again, we can consider that they are null.

More surprising and new, some high values exist between inspections associated with states 2 and 4 . However, we note that these values do not have a great influence on the cost. For example, in the case where $\mathcal{M}_{\mathrm{g}}=\{1,2,3\}$, the cost of 17.038 can also be obtained with:

$$
m_{1}=0.308, \quad m_{2}=11.398, \quad m_{3}=8.2443,
$$

or with

$$
m_{1}=0.308, \quad m_{2}=13.214, \quad m_{3}=10.946 \text {. }
$$


In the case $\mathcal{M}_{\mathrm{g}}=\{1,4\}$, the cost of 17.363 was also found using the algorithm with

$$
m_{1}=0.869, \quad m_{4}=20.685
$$

For $\mathcal{M}_{\mathrm{g}}=\{1,2,4\}$, the cost of 16.647 was also obtained with

$$
m_{1}=0.631, m_{2}=10.407, m_{4}=0.297
$$

\section{Conclusion}

We have presented a predictive preventive maintenance model (still called conditional preventive maintenance), which is applied to an initial system comprising either one component with several working states or several components each having one or more working states.

The failure rates of components are assumed to be constant in time, but in the case of several components, they may depend on the state of other components (interacting components). The lengths spent between inspections, on maintenance and on repair all have continuous distributions (i.e, they have probability density functions with respect to the Lebesgue measure).

We have given equations that allow one to calculate the stationary distribution of the system and the average cost of maintenance over an infinite horizon. This cost takes into account not only the different intervention costs, but also the costs due to immobilization of the material, by distinguishing between predicted immobilization (due to preventive maintenance) and unpredictable immobilization (due to a failure). In usual cases this cost depends only on the mean of the length of time spent during maintenance and during repairs and not on other characteristics of their distribution functions.

The precise results concerning the stationary distribution of the process constructed through the addition of supplementary variables and methods employed for calculating costs allow one to take into account other costs.

In the case where lengths of time spent between inspections follow the gamma distribution we have shown how to make numerical calculations and we have used two very different examples.

In each example, we have noted that the optimum policy for time between inspections was deterministic: the variances of the optimum distributions are so small that they may be considered to equal zero. A mathematical demonstration of this result remains a challenge.

\section{Appendix}

Let us suppose that the subset $\mathcal{H}$ is not absorbing, i.e., for any $(\eta, i, x) \in E_{1} \times \mathcal{L} \times$ $\mathbb{R}_{+}, \mathbb{P}_{(\eta, i, x)}\left(\exists t: \Phi_{t} \in \mathscr{P} \cup \mathcal{E}\right)=1$.

Let $S_{n}(n \geq 1)$ be the successive times when the process $\left(\Phi_{t}\right)$ enters a state belonging to $\mathscr{P} \cup \mathcal{B}$ :

$$
S_{1}=\inf \left(t: \Phi_{t} \in \mathscr{\rho} \cup \mathcal{E}\right\}
$$




$$
\text { for } n \geq 2, S_{n}=\inf \left\{t>S_{n-1}, \Phi_{t} \neq \Phi_{S_{n-1}}, \Phi_{t} \in \mathscr{P} \cup \&\right\} \text {. }
$$

Let $T_{1}$ be the first jump time of the process $\left(\Phi_{t}\right): T_{1}=\inf \left\{t: \Phi_{t} \neq \Phi_{0}\right\}$. Since $\mathbb{P}_{\eta}\left(\Phi_{T_{1}} \in \mathcal{M}_{g}\right)=1$ for any $\eta \in \mathscr{P} \cup \mathcal{E}$, the $\left(S_{n}\right)$ are the successive enter times into $\Phi \cup 8$.

The states belonging to $\mathscr{P} \cup \&$ are regenerative points for the process $\left(\Phi_{t}\right)$, therefore the chain $\left(\Phi_{S_{n}}\right)$ is a Markov chain taking its values in the finite space $\mathscr{P} \cup \mathcal{E}$. Let $\eta_{r}$ be a recurrent ${ }^{n}$ point for the Markov chain $\left(\Phi_{S_{n}}\right)$. We obtain immediately the following proposition:

Proposition 17: Let us suppose that the subset $\mathcal{M}$ is not absorbing and that the Markov chain $\left(\Phi_{S_{n}}\right)$ has only one recurrent class. Then there exists a regeneration state $\eta_{r} \in \mathscr{P} \cup \mathcal{E}$ such that for any $(\eta, i, x) \in E_{1} \times \mathcal{L} \times \mathbb{R}_{+}, \mathbb{P}_{(\eta, i, x)}\left(\exists t: \Phi_{t}=\eta_{r}\right)=1$.

A sufficient condition for having only one recurrent class is given in the next proposition.

Proposition 18: Let us suppose that the subset $\mathcal{H}$ is not absorbing, that the graph $\mathrm{Gg}_{\mathrm{g}}$ is irreducible and that for any $i \in \mathcal{L}$, any $x \in \mathbb{R}_{+}, \Lambda_{i}(x)>0$. Then the Markov chain $\left(\Phi_{S_{n}}\right)$ has only one recurrent class.

Hypothesis on $\Lambda_{i}$ can be weakened, but this leads to more technical proofs.

Proposition 18 is a consequence of the following lemmas. These lemmas are quite intuitive and their proofs are not difficult, but need some tedious notations, so we omit them. We also omit for each of them to recall some of the hypothesis of Proposition 18 .

Lemma 19: If there exists an arrow from $\xi$ to $\eta$ in Graph $\mathfrak{g}_{g}$, then for any $a>0$, $b>0$,

$$
\inf _{x \leq a} \mathbb{P}_{(\xi, i, x)}\left(\exists t: \Phi_{t}=\eta, X_{t} \leq x+b\right)>0 .
$$

Lemma 20: If there exists a path from $\xi$ to $\eta$ in Graph $\mathrm{g}_{g}$, then for any $a>0$, $b>0$,

$$
\inf _{x \leq a} \mathbb{P}_{(\xi, i, x)}\left(\exists t: \Phi_{t}=\eta, X_{t} \leq x+b\right)>0 .
$$

Lemma 21: Let $\eta \in \mathscr{P} \cup \&$ such that $m(\eta)>0$. Then

$$
\mathbb{P}_{(\eta, 0,0)}\left(\exists t, \exists \xi \in \mathcal{M}_{\mathrm{g}}: \Phi_{t}=\xi, I_{t}=\ell(\xi), X_{t}=0\right)=1 .
$$

Lemma 22: Let $\eta$ be a recurrent state for the Markov chain $\left(\Phi_{S_{n}}\right)$. Then there exists $\xi \in \mathcal{M}_{\mathrm{g}}$ such that

$$
\inf _{x \leq a} \mathbb{P}_{(\xi, i, x)}\left(\exists t: \Phi_{t}=\eta, I_{t}=0, X_{t}=0\right)>0 .
$$

\section{References}

[1] Cocozza-Thivent, C., Convergence de fonctionnelles de processus semirégénératifs, Prépublications de l'Equipe d'Analyse et de Matématiques Appliquées, Univ. de Marne-la-Vallée 2/00 (2000).

[2] Cocozza-Thivent, C. and Roussignol, M., A general framework for some asymptotic reliability formulas, Adv. in Appl. Prob. 32:2 (2000), 446-467. 
[3] Grall, A., Berenguer, C. and Chu, C., Optimal dynamic inspection/replacement planning in condition-based maintenance for deteriorating systems, Intern. Conf. on Safety and Reliab.-ESREL'98, Trondheim (1998), 381-388.

[4] Scarf, P.A., On the application of mathematical models in maintenance, Euro. J. of Oper. Res. 99 (1997), 493-506.

[5] Shurenkov, V.M., On the theory of Markov renewal, Th. Probab. Appl. 29 (1984), 247-265. 


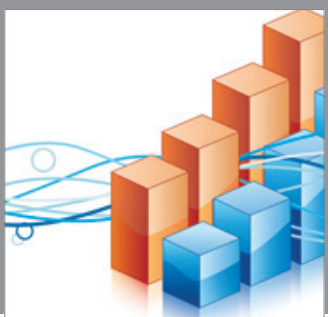

Advances in

Operations Research

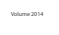

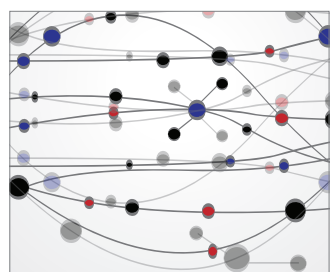

\section{The Scientific} World Journal
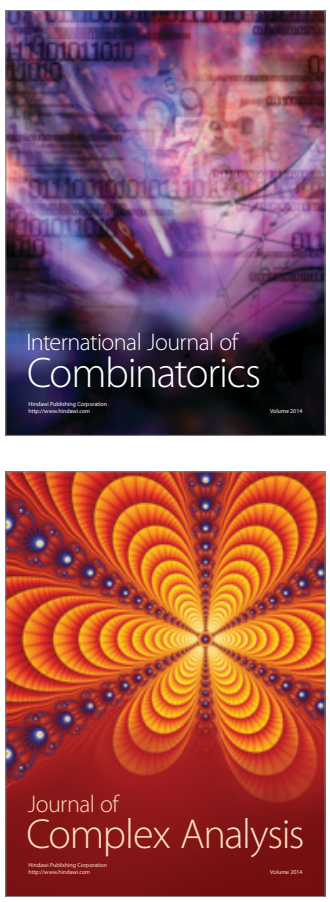

International Journal of

Mathematics and

Mathematical

Sciences
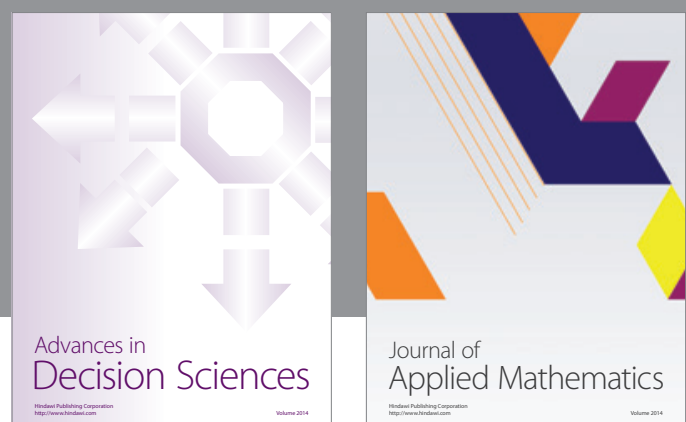

Journal of

Applied Mathematics
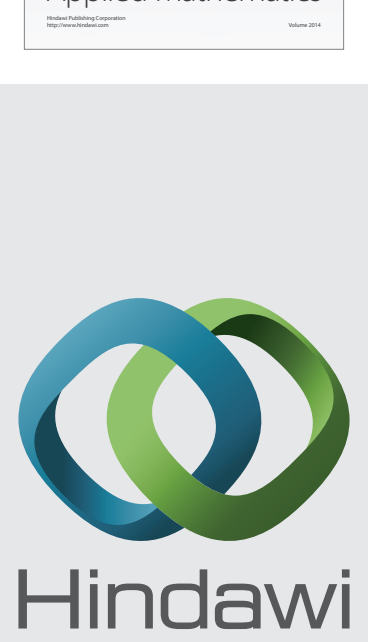

Submit your manuscripts at http://www.hindawi.com
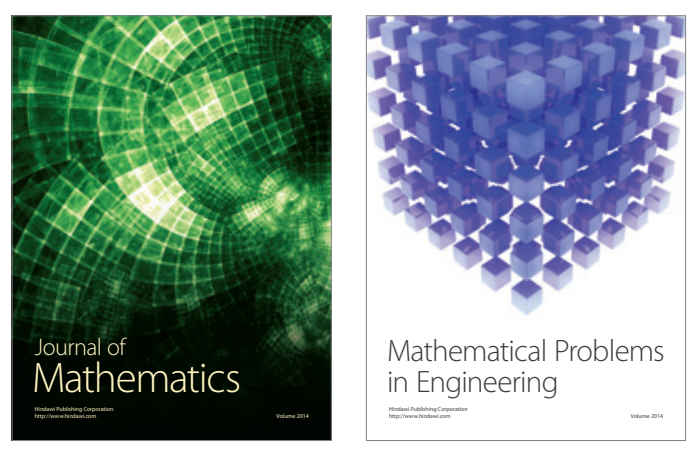

Mathematical Problems in Engineering
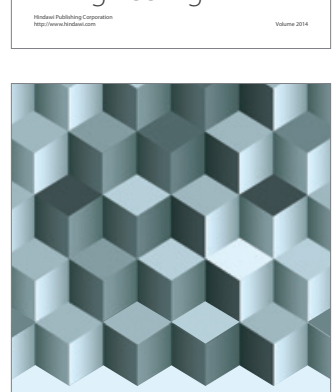

Journal of

Function Spaces
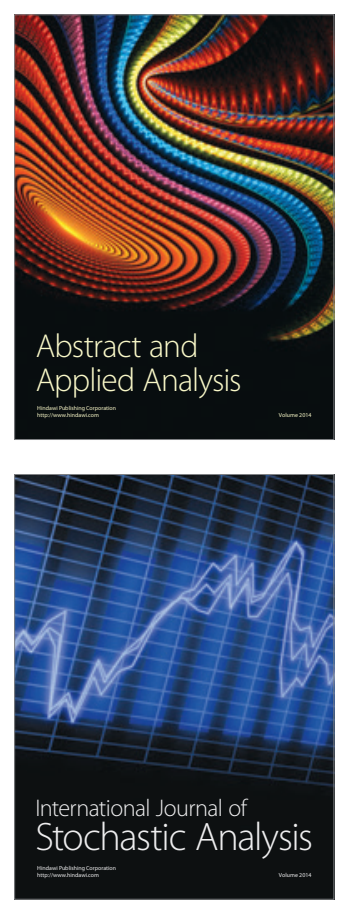

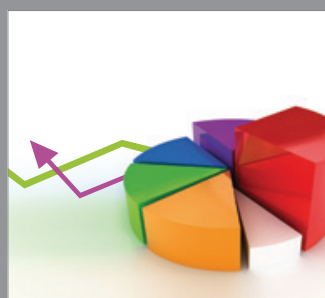

ournal of

Probability and Statistics

Promensencen
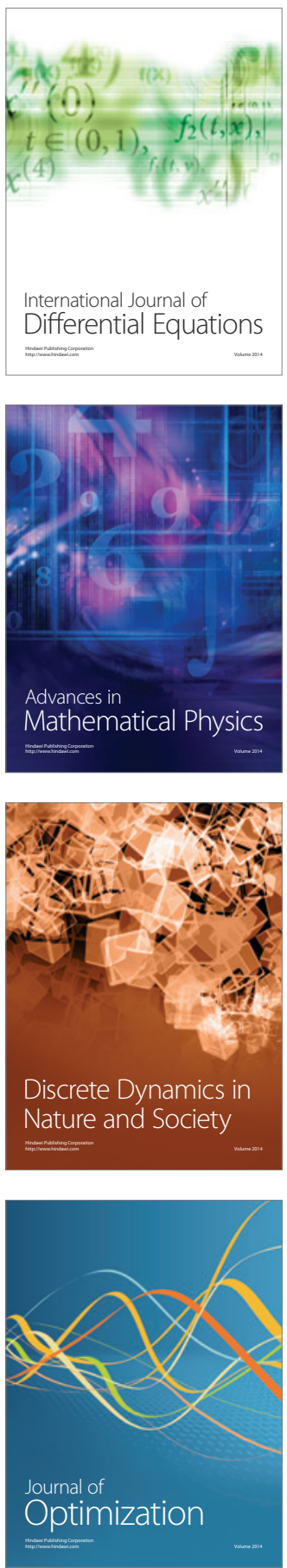